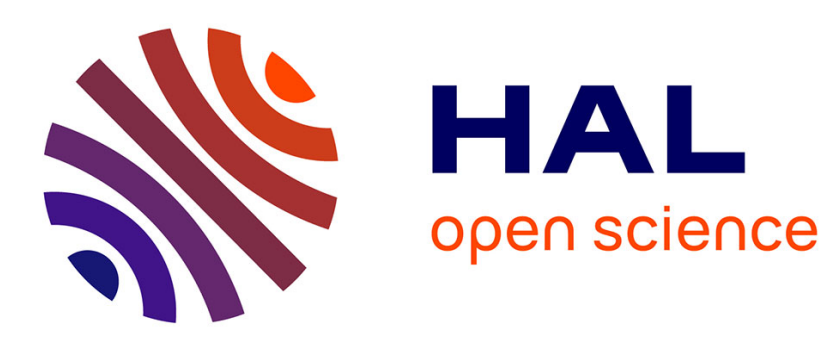

\title{
Temperature evolution of the boson peak and Debye scaling in vitreous $\mathrm{B} 2 \mathrm{O} 3$
}

\author{
Marco Zanatta, C Armellini, Aldo Fontana, F. Rossi
}

\section{To cite this version:}

Marco Zanatta, C Armellini, Aldo Fontana, F. Rossi. Temperature evolution of the boson peak and Debye scaling in vitreous B2O3. Philosophical Magazine, 2011, pp.1. 10.1080/14786435.2010.549518 . hal-00670236

\section{HAL Id: hal-00670236 https://hal.science/hal-00670236}

Submitted on 15 Feb 2012

HAL is a multi-disciplinary open access archive for the deposit and dissemination of scientific research documents, whether they are published or not. The documents may come from teaching and research institutions in France or abroad, or from public or private research centers.
L'archive ouverte pluridisciplinaire HAL, est destinée au dépôt et à la diffusion de documents scientifiques de niveau recherche, publiés ou non, émanant des établissements d'enseignement et de recherche français ou étrangers, des laboratoires publics ou privés. 


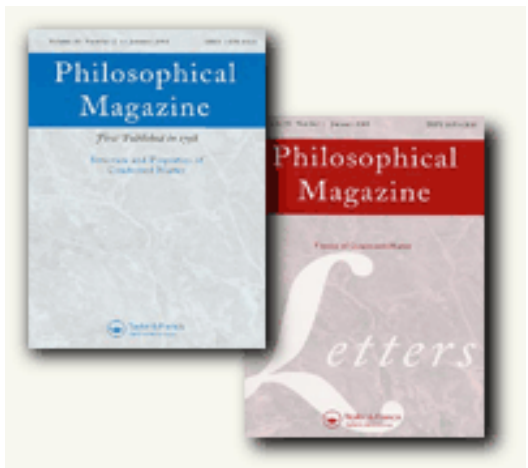

\section{Temperature evolution of the boson peak and Debye scaling in vitreous $\mathrm{B}_{2} \mathrm{O}_{3}$}

\begin{tabular}{|r|l|}
\hline Journal: & Philosophical Magazine \& Philosophical Magazine Letters \\
\hline Manuscript ID: & TPHM-10-Jun-0252.R2 \\
\hline Journal Selection: & Philosophical Magazine \\
\hline Date Submitted by the & 10 -Dec-2010 \\
\hline Complete List of Authors: & $\begin{array}{l}\text { Zanatta, Marco; Università di Trento, Dipartimento di Fisica } \\
\text { Armellini, C; IFN-CNR, CSMFO Group } \\
\text { Fontana, Aldo; university of trento, physics } \\
\text { Rossi, F.; Universita' di Trento, Fisica }\end{array}$ \\
\hline Keywords: & glass, boson peak, Raman spectroscopy \\
\hline Keywords (user supplied): & elastic medium transformation \\
\hline &
\end{tabular}

\section{SCHOLARONE $^{\text {m }}$ Manuscripts}


Philosophical Magazine

Vol. 00, No. 00, 21 December 2008, 1-5

\title{
RESEARCH ARTICLE
}

\section{Temperature evolution of the boson peak and Debye scaling in vitreous $\mathrm{B}_{2} \mathrm{O}_{3}$}

\author{
M. Zanatta ${ }^{\mathrm{a} *}$, C. Armellini ${ }^{\mathrm{b}}$, A. Fontana ${ }^{\mathrm{a}, \mathrm{c}}$, and F. Rossi ${ }^{\mathrm{a}}$ \\ ${ }^{\mathrm{a}}$ Dipartimento di Fisica, Università di Trento, I-38050 Povo, Trento, Italy; ${ }^{\mathrm{b}}$ CNR-IFN, \\ Istituto di Fotonica e Nanotecnologie, CSMFO Group, 38050 Povo, Trento, Italy; \\ ${ }^{c}$ CNR-IPCF, UOS Roma, c/o Università di Roma "La Sapienza", I-00185 Roma, Italy.
}

(December 10, 2010)

\begin{abstract}
We present a Raman scattering study of the $\mathrm{B}_{2} \mathrm{O}_{3}$ as a function of temperature. Measurements have been done from liquid helium up to the supercooled liquid state. We found that the BP shift could be completely ascribed to the temperature elastic medium transformation and we do not need the introduction of other mechanisms. Furthermore we show also in this system , that the boson peak spectral shape is invariant.
\end{abstract}

\begin{abstract}
Keywords: glasses, boson peak, elastic medium transformation, Raman light scattering
\end{abstract}

\section{Introduction}

A common feature of glasses and supercooled liquids is the presence in the vibrational density of states $g(\omega)$, of an excess of modes over the Debye level. In Raman and neutron inelastic scattering spectra it appears as a broad maximum in the so called reduced density of states $g(\omega) / \omega^{2}$, and it is known as boson peak (BP), [1-7]. Many different approaches have been proposed to explain the origin and the nature of these extra modes, but this topics are still objects of debate, [8-18].

In recent years many experimental efforts have been devoted to understand the evolution of the $\mathrm{BP}$ as a function of thermodinamical parameters like temperature $[23,24]$ and pressure [25]. Moreover, the BP has been also studied as a function of density [26-28] and time during a chemical vitrification process [29]. A general result is that, when the density is increased, no matter how, the BP position $\omega_{B P}$ shifts towards higher frequencies and it decreases its intensity. Moreover, plotting spectra corresponding to different densities as a function of $\omega / \omega_{B P}$, they collapse on a master curve $[23,27]$. This shows that the BP spectral shape is invariant: any intensity variation is only apparent and it is due to the BP shift. The explanation of the origin of this shift is now the open problem. Assuming an acoustic nature for the BP mode $[8,9,14]$, the shift can be explained by considering the elastic medium evolution i.e. the variation of the Debye frequency $\omega_{D B}[23-27,29]$. This approach has been applied to many different systems with contrasting results. As a matter of fact it provides a good scaling only in some cases [24, 26, 29] so we

*Corresponding author. E-mail: zanatta@science.unitn.it 
can conclude that elastic medium transformation does not always cover the whole story.

In this paper we present a Raman scattering study of the boson peak temperature evolution in vitreous boron oxide $\mathrm{v}-\mathrm{B}_{2} \mathrm{O}_{3}$, from the liquid helium up to the supercooled region. Boron oxide is the object of many detailed studies (e.g. references [19-22], in what follows we point out our attention on two specific points: the existence of the already mentioned master curve and the comparison between $\mathrm{BP}$ shift and elastic medium modification.

\section{Experimental details}

Vitreous $\mathrm{B}_{2} \mathrm{O}_{3}$ is a strong glass with $m \simeq 32$ and a rather low glass transition temperature, $T_{g} \simeq 576 \mathrm{~K}[30]$.

The sample was prepared starting from Aldrich boron oxide powder, purity greater than $99.995 \%$. The powder was dehydrated at $100^{\circ} \mathrm{C}$ and then melted at $1100^{\circ} \mathrm{C}$ in an alumina crucible. The homogeneous and bubble free melt was subsequently quenched in air. The obtained samples were polished in order to obtain good optical surface.

Raman scattering experiments were performed using a Jobin-Yvon U1000 double monochromator in $90^{\circ}$ geometry. As a light source it was used the $514.5 \mathrm{~nm}$ line of an argon ion laser. The spectra were acquired in a wide range of frequencies, from $-300 \mathrm{~cm}^{-1}$ to $1300 \mathrm{~cm}^{-1}$ with different resolutions. In the low frequencies region the resolution is about $2 \mathrm{~cm}^{-1}$. The measurements were performed in both $\mathrm{VV}$ and HV polarization geometries, where $\mathrm{V}$ and $\mathrm{H}$ indicate the polarizations perpendicular and parallel to the scattering plane.

\section{Results and discussion}

The first order Raman scattering intensity for a Stokes process can be written as [31]:

$$
I_{R}(\omega)=C(\omega) g(\omega) \frac{[n(\omega, T)+1]}{\omega} ;
$$

where $n(\omega, T)$ is the Bose-Einstein population factor and $C(\omega)$ is the lightvibrations coupling function. Dividing the Raman intensity for $[n(\omega, T)+1]$ and $\omega$ we obtain the so called reduced Raman intensity $I^{r e d}(\omega)$, which is proportional to the reduced density of vibrational states $g(\omega) / \omega^{2}$, namely

$$
I^{r e d}(\omega)=C(\omega) \frac{g(\omega)}{\omega^{2}} ;
$$

Reduced Raman depolarized (HV) spectra are reported in figure 1 for selected temperatures. Spectra at different temperatures are normalized to the same total area in order to be compared. High temperature spectra are characterized by the rise of a quasielastic scattering signal (QES) which slightly modifies also the BP parameters. The QES contribution has then been subtracted using the procedure described in ref. [23]. It can be seen that, when the temperature is increased, hence the density decreases, the BP peak shifts towards lower frequencies and increases its intensity. In the temperature range investigated, the position shift is $\sim 20 \%$ 


\section{Page 4 of 6}

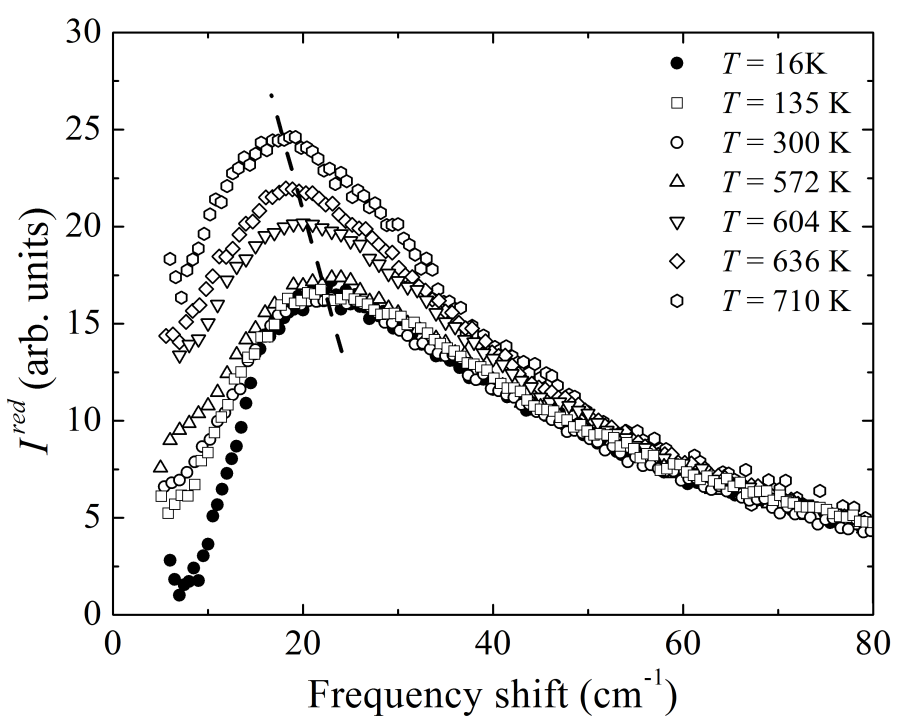

Figure 1. Reduced depolarized Raman intensity for the $\mathrm{v}-\mathrm{B}_{2} \mathrm{O}_{3}$, symbols are as in the caption; the dashed line is a guide for the eyes and it represents the temperature evolution of the BP.

We start our analysis of present data by considering the BP spectral shape by using a rescaled frequency $\nu=\omega / \omega_{s}$ and performing the variable transformation $g(\nu) d \nu=g(\omega) d \omega$. Assuming that, in the BP frequency range $C(\omega) \sim \omega,[32,33]$, the squeezed intensity can be written as:

$$
I_{s}(\nu)=I^{r e d}(\omega) \times \omega_{s}^{2}
$$

The so squeezed spectra are reported in figure 2; within the experimental uncertainty they collapse on a single master curve without any adjustable parameter. The frequency $\omega_{s}$ is defined as the frequency which allows this scaling for each spectrum. In this case $\omega_{s}=\omega_{B P}$, as it is clearly visible in the inset of figure 2 . The small differences, smaller than the uncertainty, could be ascribed to the difficulty in obtaining Raman spectra in absolute intensities.

The existence of this master curve shows that the BP evolution is ruled by its shift. To understand the origin of this feauture, we compare $\omega_{B P}$ to the elastic properties of the systems, which are described trough the Debye frequency $\omega_{D B}$. The Debye frequency has been computed using sound velocities from ref. [34], measured by Brillouin light scattering, and density from [35]. The $T$ evolution of the sound velocities is reported in the inset of figure 3 where a transverse sound wave is still visible also in the supercooled and liquid region, up to $\sim 1300 \mathrm{~K}$. This allows the use of the Debye frequency formula also above the glass transition temperature. In the explored $T$ range the density decreases of about $18 \%$. The comparison beteween $\omega_{B P}$ and $\omega_{D B}$ is reported in figure 3 : it can be seen that they exhibit the same $T$ behavior. The boson peak position scales with the Debye frequency $\omega_{B P}$, i.e. the transformation visible in the reduced density of states $g(\omega) / \omega^{2}$ can be ascribed to the elastic medium modifications. 


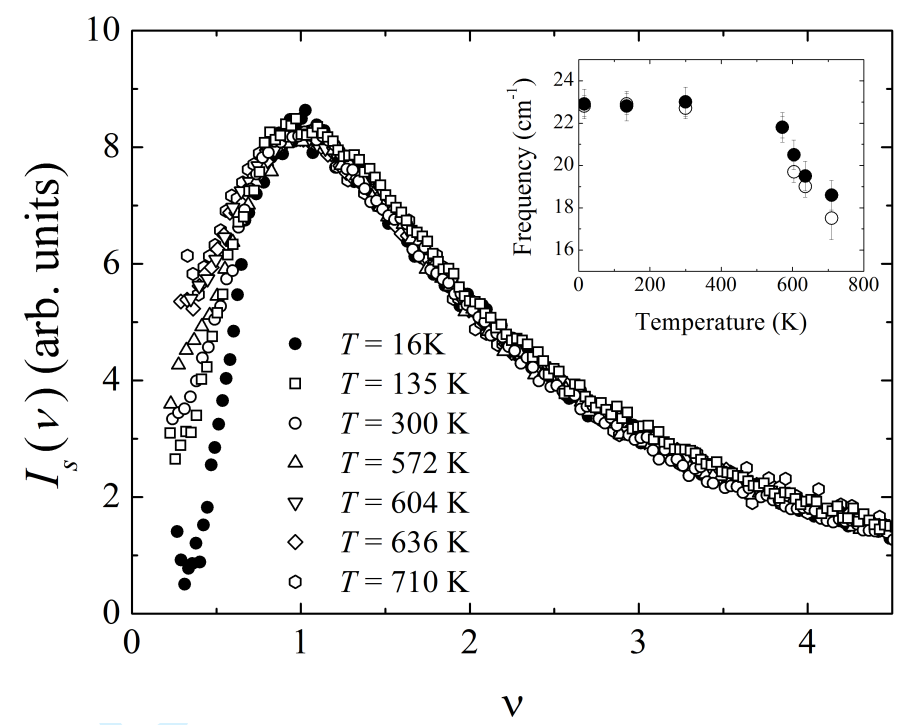

Figure 2. Master curve obtained as described in the text, symbols are as in the caption; in the inset is reported a comparison between $\omega_{B P}$ and $\omega_{s}$, respectively indicated with dots and open circles.

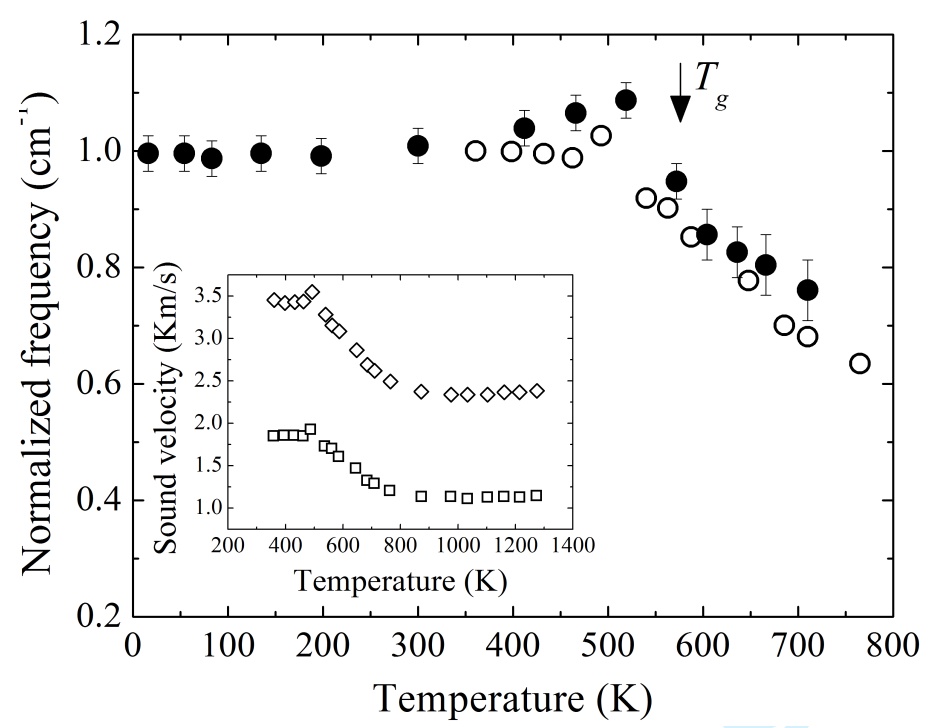

Figure 3. Comparison between $\omega_{B P}$ (dots) and $\omega_{D B}$ (open circles); longitudinal (open diamonds) and transverse (open squares) sound velocity are reported in the inset [34].

\section{Conclusions}

We investigated the temperature evolution of the boson peak in vitreous $\mathrm{B}_{2} \mathrm{O}_{3}$. We find a master curve for the BP, showing the invariance of the shape. Moreover we find also that the BP shift in this glass could be accounted using the elastic medium transformation approach. Similar studies performed in other strong systems, like vitreous silica [23, 28] and vitreous germania [27], pointed out the absence of such a scaling property, thus invocating the existence of further mechanisms that rule the BP shift. This completely different result can add a piece to understand the nature of these processes.

\section{References}

[1] E. Fabiani, A.Fontana, U. Buchenau, J. Chem. Phys. 128, 244507 (2008) and references therein. 


\section{Page 6 of 6 \\ Philosophical Magazine \& Philosophical Magazine Letters}

[2] A. Fontana, E. Moser, F. Rossi, R. Campostrini, G. Carturan, J. Non-Cryst. Solids 212, 292 (1997)

[3] K. Lipinska-kalita, A. Fontana, A. Leonardi, G. Carini, G. D'Angelo, G. Tripodo, G. Saunders, Phil. Mag. B 71, 571-581 (1995).

[4] P. Benassi, A. Fontana, W. Frizzera, M. Montagna, V. Mazzacurati, G. Signorelli, Phil. Mag. B 71 761 (1995).

[5] A.P. Sokolov, U. Buchenau, W. Steffen, B. Frick, and A. Wischnewski, Phys. Rev. B 52, R9815 (1995).

[6] S. N. Taraskin, Y. L. Loh, G. Natarajan, and S. R. Elliott, Phys. Rev. Lett. 86, 1255 (2001).

[7] W. Götze, and M. R. Mayr, Phys. Rev. E 61, 587 (2000).

[8] O. Pilla, L. Angelani, A. Fontana, J. R. Gonsalves, and G. Ruocco, J. Phys. Cond. Matt. 15, S995 (2003).

[9] O. Pilla, S. Caponi, A. Fontana, J. R. Goncalves, M. Montagna, F. Rossi, G. Viliani, L. Angelani, G. Ruocco, G. Monaco, F. Sette, J. Phys. Cond. 16, 8519 (2004).

[10] B. Ruzicka, T. Scopigno, S. Caponi, A. Fontana, O. Pilla, P. Giura, G. Monaco, E. Pontecorvo, G. Ruocco, and F. Sette, Phys. Rev. B 69, 100201 (2004).

[11] P. Benassi, S. Caponi, R. Eramo, A. Fontana, A. Giugni, M. Nardone, M. Sampoli, and G. Viliani, Phys. Rev. B 71, 172201 (2005).

[12] C. Masciovecchio, G. Baldi, S. Caponi, L. Comez, S. Di Fonzo, D. Fioretto, A. Fontana, A. Gessini, S. C. Santucci, F. Sette, G. Viliani, P. Vilmercati, and G. Ruocco, Phys. Rev. Lett. 97, 035501 (2006).

[13] W. Schirmacher, Europhys. Lett., 73(6), 892 (2006).

[14] H. Shintani and H. Tanaka, Nature Mater. 7, 870 (2008).

[15] T. S. Grigera, V. Martin-Mayor, G. Parisi, and P. Verrocchio, Nature 422, 289 (2003).

[16] D. A. Parshin, H. R. Schober, and V. L. Gurevich, Phys. Rev. B 76, 064206 (2007).

[17] B. Rufflé, D. A. Parshin, E. Courtens, and R. Vacher, Phys. Rev. Lett. 100, 015501 (2008), and references therein.

[18] E. Duval, A. Boukenter, and T. Achibat, J. Phys.: Condens. Matter 210227 (1990).

[19] A. Brodin, L. Brjesson, D. Engberg, L. M. Torell, and A. P. Sokolov, Phys. Rev. B 53, 11511 (1996).

[20] D. Engberg, A. Wischnewski, U. Buchenau, L. Brjesson, A. J. Dianoux, A. P. Sokolov, and L. M. Torell, Phys. Rev. B 58, 9087 (1998).

[21] D. Engberg, A. Wischnewski, U. Buchenau, L. Brjesson, A. J. Dianoux, A. P. Sokolov, and L. M. Torell Phys. Rev. B 59, 4053 (1999).

[22] N. V. Surovtsev, J. Wiedersich, A. E. Batalov, V. N. Novikov, M. A. Ramos, and E. Rssler, J. Chem. Phys. 113, 5891 (2000)

[23] S. Caponi, A. Fontana, F. Rossi, G. Baldi, and E. Fabiani, Phys. Rev. B 76, 092201 (2007).

[24] G. Baldi, A. Fontana, G. Monaco, L. Orsingher, S. Rols, F. Rossi, B. Ruta, Phys. Rev. Lett. 102, 195502 (2009).

[25] K. Niss, B. Begen, B. Frick, J. Ollivier, A. Beraud, A. Sokolov, V. N. Novikov, and C. Alba-Simionesco, Phys. Rev. Lett. 99, 055502 (2007).

[26] A. Monaco, A. I. Chumakov, G. Monaco, W. A. Crichton, A. Meyer, L. Comez, D. Fioretto, J. Korecki, and R. Rüffer, Phys. Rev. Lett. 97, 135501 (2006).

[27] L. Orsingher, A. Fontana, E. Gilioli, G. Carini, Jr., G. Carini,G. Tripodo, T. Unruh, and U. Buchenau, J. Chem. Phys. 132, 124508 (2010).

[28] M. Zanatta, G. Baldi, S. Caponi, A. Fontana, E. Gilioli, M. Krish, C. Masciovecchio, G. Monaco, L. Orsingher, F. Rossi, G. Ruocco, and R. Verbeni, Phys. Rev. B 81, 212201 (2010).

[29] S. Caponi, S. Corezzi, D. Fioretto, A. Fontana, G. Monaco, and F. Rossi, Phys. Rev. Lett. 102, 027402 (2009).

[30] R. Böhmer, K. L. Ngai, C. A. Angell, J. Plazek, J. Chem. Phys. 99, 4201-4209 (1993).

[31] F. L. Galeener and P. N. Sen, Phys. Rev. B 17, 1928 (1978).

[32] A. Fontana, R. Dell'Anna, M. Montagna, F. Rossi, G. Viliani, G. Ruocco, M. Sampoli, U. Buchenau, A. Wischnewski, Europhys. Letters 47, 56 (1999).

[33] A. Fontana, F. Rossi, G. Viliani, S. Caponi, E. Fabiani, G. Baldi, G. Ruocco, and R. Dal Maschio, J. Phys. Cond. Matt. 19205145 (2007).

[34] M. Grimsditch, R. Bhadra and L. M. Torell, Phys. Rev. Lett. 62, 2616 (1989).

[35] P. B. Macedo, W. Capps, and T.A. Litovitz. J. Chem. Phys. 44, 3357 (1966). 